\title{
Ambivalenzen der brasilianischen Vergangenheitspolitik und Erinnerungskultur
}

\author{
Nina Schneider
}

Am 10. Dezember 2014 veröffentlichte die brasilianische Wahrheitskommission (Commissão nacional da verdade, 2012-2014) - beinahe 30 Jahre nach der formellen Rückkehr zur Demokratie im Jahr 1985 - im Rahmen eines offiziellen Festaktes ihren Abschlussbericht. Präsidentin Dilma Rousseff, selbst ein Opfer der Diktatur, nahm den Bericht im Amtssitz der Regierung persönlich entgegen. Während sie ihre offizielle Ansprache hielt, schossen ihr Tränen in die Augen. Spontan erhoben sich die Anwesenden im Raum, um den Moment der Stille zu überbrücken - Opferfamilien, Mitarbeiter der Wahrheitskommission und Pressevertreter. Jahrzehntelang war Brasilien das einzige postautoritäre Land auf dem südamerikanischen Kontinent, welches weder eine offizielle Wahrheitskommission eingerichtet noch mutmaßliche Menschenrechtsverbrecher bestraft hat. Auch heute noch kann es Folteropfern passieren, ihre Schergen am Obststand im Supermarkt zu treffen. Die Täter des Militärregimes wurden bis heute nicht zur Rechenschaft gezogen.

Dennoch geschah etwas Erstaunliches. Als Rousseff ihre Tränen wieder unter Kontrolle hatte und ihre Rede fortsetzte, versicherte sie, dass der gesellschaftliche Pakt (der Straffreiheit) nicht gebrochen würde. Die Frau, die als Neunzehnjährige inhaftiert wurde und Elektroschocks an ihren Brustwarzen, Händen, Füßen, Oberschenkeln und ihrem Kopf erhielt, beteuerte, dass der Abschlussbericht der Wahrheitskommission kein „Rachefeldzug“ (revanchismo) sei, und sprach wiederholt von „Versöhnung“ (Wilcken 2012: 78). Damit adressierte sie die Kritiker der Wahrheitskommission und beruhigte das Militär, denn eine der Empfehlungen der Wahrheitskommission lautet, Menschenrechtsverbrecher erstmalig zur Rechenschaft zu ziehen. Kurzum: Trotz mehr als zwei Jahren Haft und ihrer Erfahrung als Folteropfer sprach sich Rousseff an diesem 10. Dezember 2014 für die Straffreiheit der Täter und für „Versöhnung“ aus.

Dieser Beitrag nimmt die Nationale Wahrheitskommission (20122014) als konkretes historisches Beispiel, um Ambivalenzen der brasilianischen Vergangenheitspolitik und Erinnerungskultur herauszuarbeiten. Dem Thema „Geschichte wird gemacht“ folgend, analysiere ich das wi- 
dersprüchliche Verhalten von Akteuren, die in Brasilien einen wesentlichen Beitrag zur Blockade bzw. Entwicklung der brasilianischen Vergangenheitsaufarbeitung geleistet haben. In früheren Publikationen habe ich bereits argumentiert, dass der Staat kein homogenes Gebilde ist, sondern unterschiedliche Akteure eine Aufarbeitung gefordert bzw. behindert haben (Schneider 2014). Zudem habe ich in einer weiteren Studie untersucht, welche Gesellschaftsgruppen die Einführung der Wahrheitskommission erstritten bzw. bekämpft haben (Schneider 2013). Dieser Beitrag analysiert nun das widersprüchliche Verhalten ausgewählter staatlicher, kultureller und gesellschaftlicher Akteure und argumentiert, dass die Erinnerungsblockaden in Brasilien komplexere Hintergründe haben als nur einen passiven Staat und eine verfehlte Vergangenheitspolitik. Ja, der Staat hat jahrzehntelang eine Aufarbeitung behindert, aber eine tiefergehende Analyse der Ambivalenzen zeigt, dass die Blockade komplexere Hintergründe hat und nichtstaatliche Akteure und Medien gleichfalls in der Verantwortung stehen. Die zwei Kernthesen lauten: Erstens, staatliche Vergangenheitspolitik ist selbst umkämpfte Geschichtspolitik. Sie ist heterogen, dynamisch und potentiell widersprüchlich (wie schon das eingangs beschriebene Verhalten Rousseffs während des Festaktes des Abschlussberichtes gezeigt hat). Zweitens, Geschichte wird neben offiziellen Geschichtsschreibern auch von weiteren Akteuren gemacht: Kunst- und Kulturschaffenden, Medien und der Gesellschaft.

Methodisch bedeutet dies, dass eine einseitige Betrachtung der staatlichen Geschichtspolitik zu kurz greift. Sie wird erst aussagekräftig, wenn sie mit der Geschichtskultur einer Gesellschaft gemeinsam gedacht wird (Vergangenheitspolitik und Erinnerungskultur). Sowohl eine Erweiterung des Quellenkorpus als auch eine multidisziplinäre Herangehensweise tragen zu einem vertieften Verständnis bei. Nichtstaatliche Quellen wie etwa Kunst- und Kulturprodukte und Meinungsumfragen können mit offiziellen Quellen (Gesetze, Regierungsberichte etc.) trianguliert werden und ein umfassenderes Bild schaffen. Während die meisten Studien über Vergangenheitsbewältigung entweder einseitig staatliche Initiativen untersuchen bzw. kulturwissenschaftliche Studien sich oft auf die Analyse einzelner Kulturprodukte begrenzen, ohne den politischen Kontext miteinzubeziehen, möchte dieser Artikel einen Beitrag zu einer holistischen Analyse leisten und die staatliche, kulturelle und gesellschaftliche Ebene der Vergangenheitsaufarbeitung zusammenbringen.

Der Begriff Vergangenheitspolitik bezieht sich hier eng auf staatliche erinnerungspolitische Maßnahmen: Entschädigungszahlungen, offizielle Entschuldigungen, staatlich finanzierte Erinnerungsorte und -projekte, 
Wahrheitskommissionen und Gerichtsurteile. Es handelt sich um Maßnahmen „,von oben“. ${ }^{1}$ Im Gegensatz dazu umfasst der Oberbegriff Erinnerungskultur weitere Akteure: Kulturschaffende, zivilgesellschaftliche Organisationen, Privatleute, im Grunde genommen alle gesellschaftlichen Akteure, die auf verschiedenste Weise aktiv ein historisches Ereigniss erinnern. Die Erinnerungsformen können eine ästhetische, politische oder kognitive Form annehmen (Cornelissen 2012). Im Gegensatz zum Begriff Geschichtskultur liegt der Schwerpunkt des Begriffes Erinnerungskultur auf dem instrumentellen Gebrauch der Vergangenheit für die Gegenwart (Rüsen 1994; Cornelissen 2012). In Anlehnung an Jan Assmann (1999) und Aleida Assmann (1999; Assmann/Frevert 1999: 35-50) beschreibt Erinnerungskultur hier ein dynamisches und historisch kontingentes $\mathrm{Zu}-$ sammenspiel unterschiedlicher (oftmals antagonistischer) zivilgesellschaftlicher und staatlicher Akteure. Erinnerungskultur bezieht sich immer auch auf Akteure und soziale Praktiken „von unten“ und schließt mit ein, wie eine Gesellschaft auf vergangenheitspolitische Maßnahmen oder deren Abwesenheit reagiert.

Um folgende zentrale Fragestellungen - Welche Akteure haben die Vergangenheitspolitik/Erinnerungskultur gefördert bzw. blockiert? Welche Widersprüche können wir festmachen, die diese Blockade erklären? zu beantworten, werden hier zunächst das Militärregime und die vergleichsweise spärliche und späte Vergangenheitspolitik vorgestellt. Zwei widersprüchliche Phänomene werden diskutiert: Erstens haben Opfer innerhalb des Staatsapparates eine umfassende Vergangenheitspolitik eher blockiert als gefördert. Zweitens haben selbst die Regierungen der Arbeiterpartei (Partido dos trabalhadores, PT), eine Partei welche besonders viele Opfer zählt und sich laut Parteiprogramm mit Menschenrechten solidarisiert, keine Wende in der offiziellen Vergangenheitspolitik gebracht. Der dritte Abschnitt wendet den Blick auf nichtstaatliche Akteure: sowohl auf Kunst- und Kulturschaffende als auch auf die brasilianische Gesellschaft im Ganzen. Er beleuchtet den ambivalenten Beitrag nichtstaatlicher Akteure zur brasilianischen Erinnerungskultur. Der letzte Teil bietet einen Ausblick und erörtert, wovon es abhängen wird, ob die Empfehlungen der brasilianischen Wahrheitskommission umgesetzt werden.

1 Diese enge staatliche Definition hat den Vorteil, dass sie sich klarer vom Begriff Erinnerungskultur unterscheidet. Denkbar sind aber auch weitere Definitionen, die nicht-staatliche Maßnahmen einschließen (vgl. Eser/Peters i. E.). 
Nina Schneider

Hintergrund: das brasilianische Militärregime und der schwierige Umgang mit der Vergangenheit

Als das brasilianische Militär 1964 die Macht ergriff, wurde es von weiten Teilen der Ober- und Mittelschicht zunächst gefeiert. Sogleich wurde jedoch jegliche politische Opposition bekämpft. Das Regime entzog politischen Gegnern sowohl die Freiheitsrechte als auch das aktive und passive Wahlrecht und führte ein Zweiparteiensystem ein. Obgleich bereits seit 1964 gefoltert wurde, verschärfte sich ab 1969/70 die Repression mit der Einführung eines landesweiten Repressionsapparates. Politische Oppositionsgruppen wurden verfolgt, ins Exil getrieben, eingesperrt, gefoltert und im schlimmsten Fall ermordet. Zudem führten die Militärs die systematische Pressezensur ein. 1985 kehrte Brasilien zur formellen Demokratie zurück. Das Mehrparteiensystem und die Gewaltenteilung wurden restauriert.

Eine Geschichte der Blockade oder der Frustration - so könnte man Brasiliens Umgang mit dem Regime seit 1985 bezeichnen (zumindest aus der Sicht der Hinterbliebenen und des modernen internationalen Menschenrechts). ${ }^{2}$ Carlos Fico (2012: 25, 32 f.) bezeichnet die Transition daher als „unvollendet“ (transição inconclusa) und gekennzeichnet durch „Frustration“. Eine ähnliche Einschätzung liefert Anthony Pereira (2005: 172), der konstatiert: „Transitional justice in Brazil was minimal.“

Durch das vom Regime selbst proklamierte Amnestiegesetz von 1979 war eine juristische Aufarbeitung der Diktatur versperrt. Es schließt eine Verurteilung von damaligen Menschenrechtsverbrechern innerhalb des Staatsapparates aus. Die brasilianische Richtervereinigung (OAB) klagte 2008 gegen das Amnestiegesetz vor dem Bundesverfassungsgericht, aber die Klage wurde 2010 abgelehnt (Schneider 2011b). Politische Maßnahmen blieben ebenfalls aus. So wurde das Militär, die Polizei und das Gerichtswesen weder reformiert noch kam es zu Entlassungen. Insgesamt beginnt der brasilianische Staat gerade erst, die Diktatur ernsthaft „aufzuarbeiten“. 3

Die wichtigsten staatlichen Aufarbeitungsmechanismen waren ein zweistufiges Entschädigungsprogramm für die Opfer (beginnend 1995,

2 Siehe zur brasilianischen Transition Brito (2001); Pereira (2005); Abrão/Torelly (2011); Schneider (2011a); sowie Mezarobba (2013).

3 Der Begriff „Aufarbeitung“ ist in der historischen Forschung zu Recht problematisiert worden; doch mangelt es an Alternativkonzepten. Der Begriff „Aufarbeitung" umfasst hier sowohl staatliche als auch private Maßnahmen, welche darauf abzielen, einen konstruktiven Umgang mit einer gewaltvollen Vergangenheit zu fördern und ein Verdrängen zu verhindern. 
ausgeweitet 2002), eine staatliche Erinnerungspolitik (seit 2006) sowie kürzlich die Nationale Wahrheitskommission (2012-2014). Auf massiven Druck von Opferverbänden und nationalen und internationalen Organisationen erließ die Fernando H. Cardoso-Regierung 1995 das „Gesetz der Verschwundenen“, welches erstmals offiziell anerkannte, dass der brasilianische Staat für den Tod von 136 Bürgern die Verantwortung trägt und einen Anspruch auf Entschädigungszahlungen begründete (Mezarobba 2007; SEDH 2007: 17 ff., 48). Zudem wurde eine staatlich organisierte Aufklärungskommission eingesetzt, die sogenannte Spezialkommission (Comissão Especial Sobre Mortos e Desaparecidos Políticos, hiernach: CEMDP).

Als zweiten wesentlichen Schritt richtete der Staat im Jahr 2002 per Dekret die sogenannte Amnestiekommission als Unterorgan des Justizministeriums ein, die seither ein erweitertes Entschädigungsprogramm für Folteropfer, Zwangsentlassene und Exilierte (vorher waren es nur Todesopfer) ausführt. Seit 2002 sind 74.000 Anträge bei der Amnestiekommission eingegangen; etwa ein Viertel der Antragsteller erhielt Entschädigungszahlungen (Abrão/Torelly 2011: 443-485; Ministério da Justiça 2015). Ferner hat die brasilianische Regierung seit 2006 zahlreiche Erinnerungsprojekte initiiert einschließlich Wanderausstellungen, Diskussionsrunden, Denkmälern und Lehrerfortbildungen (Memórias Reveladas 2009; Schneider 2011a; Atencio 2014). Von zentraler Bedeutung war der Abschlussbericht der CEMDP mit dem Titel „Recht auf Erinnerung“ (Direito à memória), die erste systematische Aufklärungsstudie seitens des Staates (SEDH 2007).

Zusammenfassend kann festgestellt werden, dass alle vergangenheitspolitischen Maßnahmen eine Antwort des Staates auf den unermüdlichen Druck der Opferfamilien und ihrer Unterstützer waren. ${ }^{4}$ Die Entschädigungszahlungen waren wohl die wichtigste vergangenheitspolitische Maßnahme. ${ }^{5}$ Wenngleich die Wahrheitskommission ebenfalls ein historisch bedeutsamer Moment war, ist sie vor allem von den Opferfamilien, aber auch von Menschenrechtsorganisationen und Staatsanwälten vielfach kritisiert worden (ISER 2013; Schneider 2013). Wenn der Staat also jahrzehntelang eine Aufarbeitung behindert hat, so analysiert der nun folgende Abschnitt einen bemerkenswerten Widerspruch: Selbst Opfer der Militär-

4 Zahlreiche Studien haben dies belegt vgl. Santos (2015), Mezarobba (2007, 2014).

5 Die Amnestiekommission, die das Entschädigungsprogramm selbst ausführt, bezeichnet die Programme als den „Dreh- und Angelpunkt“ der brasilianischen Aufarbeitung (Abrão/Torelly 2011). 
Nina Schneider

diktatur im Staatsdienst haben eine aktive Vergangenheitspolitik, behindert und gerade die Arbeiterpartei, die aus der Oppositionsbewegung zum Regime entstanden ist, hat zahlreiche Maßnahmen blockiert oder abgemildert.

Widersprüchliche Politik staatlicher Akteure

Nachdem die drei ersten zivilen Präsidenten die Diktatur ignoriert hatten, war Fernando H. Cardoso (1995-2003) das erste brasilianische Staatsoberhaupt, welches vergangenheitspolitische Maßnahmen ergriff. Eine genaue Analyse zeigt jedoch das widersprüchliche Verhalten Cardosos. Das „Gesetz der Verschwundenen“ von 1995 war lediglich eine Antwort des Staates auf den massiven Druck von Opferverbänden und sowohl nationalen als auch internationalen Menschenrechtsorganisationen.

Cardoso selbst wurde 1975 kurzzeitig von den Militärs gefangen genommen. Wenngleich ihm persönlich das Folterschicksal erspart blieb, so beschreibt er in seiner Autobiographie (Cardoso 2006), wie er zwangsweise Zeuge davon wurde, wie Regimegegner im Nebenzimmer gefoltert wurden. Als Soziologieprofessor mit (damals) marxistischem Hintergrund und zentraler Referenzpunkt der Dependenztheorie, verbrachte Cardoso viele Jahre im Exil. ${ }^{6}$

Dass Cardoso sich trotz seiner Repressionserfahrung weigerte, die Gräueltaten des Staates aufzuklären, ist ihm von den Opferfamilien verübelt worden. Vergeblich bemühten sie sich, ihn zu einer offiziellen Entschuldigung des Staates zu bewegen. Als sie mit ihrer Forderung innenpolitisch scheiterten, baten sie internationale Menschenrechtsorganisationen wie Amnesty International (AI) und Human Rights Watch (HRW) um Hilfe (Mezarobba 2007: 50 ff.). Pierre Sane, der damalige AI-Vertreter, traf sich persönlich mit Cardoso, um Druck auf ihn auszuüben, sich endlich offiziell für die Menschenrechtsverbrechen des Staates zu entschuldigen. Als die Entschuldigung ausblieb, attackierte Sane Cardoso öffentlich. HRW forderte die brasilianische Regierung auf ähnliche Weise auf, staatliche Menschenrechtsverbrechen anzuerkennen, aufzuklären und die Opfer zu entschädigen (Souza 1995: 11).

Als die Entschuldigung 1995 aufgrund des nationalen und internationalen Druckes endlich kam, war das Hauptproblem nicht einmal, dass sie bescheiden, geradezu klammheimlich präsentiert wurde (die Nachricht

6 Seine Präsidentschaft (1995-2003) war von einer neoliberalen Politik gekennzeichnet, z. B. privatisierte er zahlreiche Unternehmen. 
schaffte es kaum in die Tageszeitung; Schneider 2014: 16 f.). Hauptproblem war, dass das Gesetz nur ein halbherziges Zugeständnis war. In der Praxis wurde die Arbeit der CEMDP von der Regierung eher behindert als unterstützt (Mezarobba 2007; SEDH 2007: 33 f.). Der Kommission fehlten neben Zugriffsrechten (z. B. Archivzugriff, das Recht, Zeugen vorzuladen) auch personelle und finanzielle Ressourcen. Während Cardoso Gründer und Mitglied der Partei der Brasilianischen Sozialdemokratie (PSDB) ist, einer Partei, die ihren Ursprung in der Mitte hatte, aber heutzutage dem konservativen Mitte-Rechts-Lager zugeordnet wird, so ist es noch erstaunlicher, dass selbst die weiter links stehende Arbeiterpartei (PT) nach ihrem Wahlsieg im Jahr 2002 nicht radikal mit dem autoritären Regime brach.

Viele Mitglieder der Regierung Lula waren in den regimekritischen Studenten- oder sogar Guerillabewegungen aktiv. Laut einer Studie von Maria Celina D’Araújo (2010) waren 49 Prozent von Lulas Ministern im politischen Untergrund aktiv. Präsident Lula (2003-2010) selbst war in den späten 1970ern und frühen 1980ern (also noch während der Diktatur) im Ballungszentrum São Paulos zur Führungsfigur der Gewerkschaften avanciert und verkörperte den prominentesten Gegner des Regimes (Alves 1985: 203, 209). Seine Karriere fußte auf seiner Rolle als Widerstandskämpfer, die ihm Anerkennung und Legitimation einbrachte. Am Ende seiner zweiten Legislaturperiode hatte Lula zudem die höchsten Zustimmungsraten, die ein brasilianischer Präsident je hatte; im Januar 2010 lag Lulas persönliche Zustimmungsrate bei 84 Prozent (Guerreiro 2009).

Trotz seiner regimekritischen Biographie und seiner historischen öffentlichen Anerkennung ließ sich Lula im Dezember 2009 vom damaligen Verteidigungsminister und der Militärführung erpressen, als es um die Einrichtung der Wahrheitskommission ging. Die Militärführung drohte ihm damit, ihr Entlassungsgesuch einzureichen, wenn der Gesetzestext der Wahrheitskommission nicht geändert würde. Anstatt sich zu widersetzen, fügte sich Präsident Lula dem militärischen Druck und veränderte den Gesetzestext entscheidend. Während im Originalvorschlag beispielsweise von Menschenrechtsverletzungen „im Kontext von politischer Repression“ die Rede war, ersetzte der neue Gesetzesvorschlag diesen Passus mit den Worten „in einem Kontext des politischen Konflikts“ (Schneider 2011c). Damit blieb unausgesprochen, um welche Menschenrechtsverbrecher es sich handelte: Agenten der staatlichen Repressionsorgane oder Mitglieder der militanten Opposition. Diese scheinbar lapidare Textänderung zeigt, dass sich selbst Opfer des Regimes, welche an die Regierung gelangen, noch immer nicht dazu bekennen wollen, dass der brasilianische 
Nina Schneider

Staat zwischen 1964 und 1985 eine Politik der systematischen Repression betrieben hatte.

Lula, der eingesperrt, aber nicht gefoltert wurde, ist nicht das einzige Mitglied der Arbeiterpartei, das eine aktive Vergangenheitspolitik paradoxerweise unterbunden hat. Neben der heutigen brasilianischen Präsidentin Rousseff ist auch der ehemalige Menschenrechtsminister Vannuchi verhaftet und gefoltert worden. Paulo Vannuchi hat seit seiner Ernennung zum Menschenrechtsminister im Jahr 2005 eine aktive Rolle in der brasilianischen Vergangenheitspolitik gespielt, aber auch sein Verhalten ist voller Widersprüche. Vannuchi wurde als einundzwanzigjähriges Mitglied der Guerillabewegung Nationale Befreiungsaktion (Ação Libertadora Nacional, ALN) gefangen genommen. Er überlebte fünf Jahre Gefängnis und brutale Folter (Schneider 2011c). Sein Cousin, Alexandre Leme Vannuchi, bekam 1973 tragische Prominenz als das erste bekannte Todesopfer des Regimes, welches zu Massenprotesten führte. Zwischen 1980 und 1985 soll Paulo Vannuchi einer der (anonymen) Autoren des Brasil Nunca Mais-Projektes gewesen sein. Zudem hat er seit Beginn die Arbeiterpartei mitaufgebaut, managte die Öffentlichkeitsarbeit und war seit 1989 führender Berater von Lulas Wahlkampagnen.

In vielen Situationen hat Vannuchi sich eindeutig für eine aktive Vergangenheitspolitik eingesetzt. Neben den seit 2006 eingeführten erinnerungspolitischen Maßnahmen hat sich Vannuchi in der Öffentlichkeit für die Wahrheitskommission und in einzelnen Situationen sogar für die Bestrafung der Täter ausgesprochen. Im Streit um die Gesetzesvorlage zur Errichtung der brasilianischen Wahrheitskommission beispielsweise argumentierte er öffentlich, dass Versöhnung nicht einfach das Verdrängen der Vergangenheit bedeuten könne. Für seinen vergangenheitspolitischen Einsatz ist er von der konservativen brasilianischen Presse persönlich und aggressiv attackiert worden (Schneider 2011a: 1 f.).

Dennoch unternahm Vannuchi einen widersprüchlichen Schachzug, als der brasilianische Staat im Jahr 2010 vom interamerikanischen Gerichtshof für Menschenrechte (IACHR) der Organisation Amerikanischer Staaten verklagt wurde. Anfang der 1990er Jahre hatten Opferfamilien mit Hilfe des Center for Justice and International Law (CEJIL) eine Sammelklage gegen den brasilianischen Staat eingereicht, welche beinahe zwei Jahrzehnte später - im Jahr 2010 - zur Urteilsverkündigung führte. ${ }^{7}$ Der IACHR

7 Die Organisation CEJIL fordert die optimale Ausnutzung des interamerikanischen Systems und unterstützt diese auf vielfältige Weise (z. B. finanziell oder durch die juristische Beratung von Opfergruppen), http://cejil.org (Stand: 2. Mai 2015). 
verurteilte den Staat Brasilien, gegen internationales Menschenrecht verstoßen zu haben, da Brasilien die Verbrecher des Militärregimes noch immer nicht bestraft habe, und gab den Opferfamilien somit Recht. Als Regierungsvertreter reiste Vannuchi damals zum IACHR. Anstatt das Urteil anzunehmen und dessen Umsetzung einzuleiten (der Vorwurf lautete immerhin Brasilien, breche internationales Menschenrecht), bat Vannuchi im Auftrag der Lula-Regierung darum, die Anklage fallen zu lassen, und spendete nach seinem Besuch eine hohe Geldsumme an den Gerichtshof. ${ }^{8}$

Insgesamt ergaben Interviews im Jahr 2012, dass viele Menschenrechtsaktivisten, darunter viele Folteropfer, sowohl von der Regierung Lula als auch Rousseff enttäuscht sind. Alle demokratischen Präsidenten hätten den Opferfamilien eine offizielle Audienz verwehrt, in der sie ihre Anliegen hätten vortragen können (Schneider 2014). Wenngleich die Präsidenten Cardoso, Lula und Rousseff den Opfern kleine Konzessionen machten, hat keiner von ihnen den Mut bewiesen, klar mit der Militärvergangenheit zu brechen.

Der ambivalente Beitrag von Kulturschaffenden und der brasilianischen Gesellschaft

Im Gegensatz zur späten und vergleichsweise bescheidenen Vergangenheitspolitik des Staates kamen seit den 1990er Jahren zahlreiche Kunstund Kulturprodukte auf den Markt, welche die Militärdiktatur thematisierten. Kinofilme, Telenovelas und Bücher (speziell das Genre der Memoiren) waren die ersten Medien, die einen gesellschaftlichen Diskurs über die Militärdiktatur anbahnten. Bereits in den 1990er Jahren, als der Staat Menschenrechtsverbrechen noch immer verschwieg, wurden Telenovelas über die Diktatur zu Quotenschlagern (Atencio 2011). Viele Brasilianer erfuhren erstmals durch Vorabendserien, dass während der Diktatur gefoltert wurde, denn zuvor waren diese Informationen entweder staatlich zensiert oder von der Regierung abgestritten worden. Neben den Telenovelas avancierten Memoiren von ehemaligen Guerillakämpfern zu Bestsellern, von denen viele später auch verfilmt wurden. Ein Klassiker ist Fernando Gabeiras „Was ist los, Kamerad?“ (O que é isso, companheiro?, 1988). Das fiktive Guerillatagebuch wurde 1997 vom brasilianischen Regisseur

8 Die Autorin dankt ihren Interviewpartnern Crimeia de Almeida Teles, Edson Teles und der Staatsanwältin und heutigen Präsidentin der Spezialkommission Eugênia Gonzaga für diese Information (Interviews 2012). 
Bruno Barreto verfilmt und in der Kategorie „bester ausländischer Film“ für den Oscar nominiert.

Wenngleich Kunst und Kultur für die Erinnerungskultur der Militärdiktatur von großer Bedeutung waren, problematisieren Schneider/Atencio (2015) die ambivalente Rolle vieler dieser Kulturprodukte. Eine genaue Analyse kultureller Erinnerungsmedien zeigt, dass die Mehrzahl der Filme und Seifenopern das Regime nicht kritisch reflektiert, sondern oftmals zur Vergessenheit anstatt zu einer aktiven, kontroversen Erinnerungskultur beitrug. Das offensichtliche kulturelle Interesse der brasilianischen Bevölkerung am Militärregime steht also nur auf den ersten Blick im Widerspruch zum öffentlichen Desinteresse an politischen und juristischen Initiativen. ${ }^{9}$ Der Widerspruch löst sich auf, wenn man berücksichtigt, dass viele dieser Kulturprodukte die Diktatur zwar thematisieren, aber romantisieren und verzerren und damit nicht zu einer kritischen Auseinandersetzung einladen. Die kulturelle Verklärung der Diktatur und das gesamtgesellschaftliche Desinteresse an den Menschenrechtsverbrechen der Diktatur verstärken sich somit gegenseitig (Schneider/Atencio 2015).

Paradoxerweise haben sich auch viele kulturschaffende Ex-Guerillakämpfer explizit gegen die Aufhebung der Amnestie ausgesprochen. Ein gutes Beispiel ist der Ex-Guerillakämpfer und Bestsellerautor Fernando Gabeira, der sich mehrfach öffentlich für Straffreiheit der Täter aussprach. Wie Atencio (2014) darlegt, hat Gabeira, der nach der Diktatur zu einem wichtigen Politiker avanciert ist und eine Führungsrolle bei den brasilianischen Grünen gespielt hat, in Parlamentsdebatten, Presseberichten und Interviews wiederholt seine Meinung gegen eine Bestrafung öffentlich kundgetan. Damit reiht er sich ein in die Liste der Akteure, die das Versöhnungsnarrativ der Amnestie von 1979 bestärkt haben. Ein weiteres Beispiel für die Reproduktion des brasilianischen Versöhnungsdiskurses ist der Brasil Nunca Mais-Bericht (BNM 1985). ${ }^{10}$ Der BNM war der erste systematische, nicht-staatliche und ist bis heute einer der wichtigsten Berichte über grobe Menschenrechtsverletzungen während des Regimes. Er gilt als Informationsgrundlage des Abschlussberichtes der Wahrheitskommission. Der BNM wurde 1985, als Brasilien nach einundzwanzig Jahren zu einem zivilen Präsidenten zurückkehrte, vom Erzbischof Dom Evaristo Arns herausgegeben. Eine schmale Buchversion des BNM wurde sofort zum Bestseller. Eine Diskursanalyse zeigt, dass der BNM zwar sys-

9 Beispielsweise fehlen in Brasilien Massenproteste für die Bestrafung der Täter wie etwa in Argentinien.

10 Der Originalbericht umfasst sechs Volumen und ist seit Juli 2013 komplett digitalisiert (BNMD 2013). 
tematische Foltermethoden erstmals im Rahmen einer breiten Öffentlichkeit denunzierte, aber gleichzeitig auch das Versöhnungsnarrativ des Amnestiegesetzes bediente. In seiner jüngsten Studie verweisen Teles/Quinalha (2015) auf folgenden Passus im BNM-Bericht (Arquidiocese 1985: 26; meine Übersetzung): „Wir [das BNM-Projekt] sind in keinster Weise durch irgendeine Art von Revanche animiert. Auf der Suche nach Gerechtigkeit war das brasilianische Volk niemals von Rachegelüsten angetrieben.“

Insgesamt lässt sich feststellen, dass breite Teile der brasilianischen Gesellschaft den Diskurs der Straffreiheit und der Versöhnung mittragen. Die Opferfamilien und ihre Helfer scheinen eine gesellschaftliche Minderheit zu sein. Zahlreiche Leserbriefe bezeugen, dass weite Teile der Bevölkerung den Versöhnungsdiskurs der Amnestie und die darin besiegelte Straffreiheit befürworten. Eben dieses Versöhnungsnarrativ bediente die brasilianische Präsidentin Rousseff im Dezember 2014, als sie von „Versöhnung“ sprach und beschwichtigte, dass der Abschlussbericht kein „Rachefeldzug“ sei.

Geschichtspolitik und Erinnerungskultur nach der Wahrheitskommission

Inwieweit der Abschlussbericht mittelfristig zu einer aktiveren Geschichtspolitik und einer engagierten gesamtgesellschaftlichen Erinnerungskultur führen wird, hängt von vielen Akteuren ab. Der Bericht (CNV 2014) enthält 29 Empfehlungen, wobei sich die meisten auf die Bestrafung der Menschenrechtsverbrecher beziehen. Die ersten drei und wichtigsten lauten: (1) Das Militär soll seine institutionelle Verantwortung eingestehen; (2) die Personen, die Verbrechen gegen die Menschlichkeit begangen haben, sollen bestraft werden; und (3) der Staat soll von den Tätern Geld zurückfordern, welches er als Entschädigung an die Opfer gezahlt habe. Weiterhin empfiehlt die Kommission, dass der Sicherheitsapparat reformiert werde, um die gegenwärtige Folterpraxis (vor allem gegenüber der armen schwarzen Bevölkerung) zu verhindern. Angesichts der fortbestehenden interessenspolitischen Gräben wird die offizielle Geschichtspolitik auch in Zukunft stark umkämpft, heterogen und dynamisch bleiben. Dennoch ist es möglich, wichtige Einflussfaktoren zu benennen, die diese Dynamik beeinflussen werden.

Wenngleich - wie gezeigt - die Arbeiterpartei mit ihren zahlreichen Mitgliedern aus der Widerstandsbewegung erstaunlich wenige vergangenheitspolitische Maßnahmen ergriff, so kommt aktuell erschwerend hinzu, 
dass sowohl die PT als auch Dilma Rousseff persönlich aus zwei Gründen politisch geschwächt sind. Erstens muss Dilma nach den turbulenten Wahlen im Herbst 2014 mit einem sehr konservativen Kongress regieren, der ihre Projekte blockieren kann. Zweitens ist der Start in die zweite Legislaturperiode von einem massiven Korruptionsskandal der Ölfirma Petrobras überschattet, der Massenproteste ausgelöst hat und der Präsidentin extrem niedrige Popularitätswerte beschafft hat (im April 2014 lediglich 13 \%; Fleischer 2015a). Auch in dieser Legislaturperiode (2015-2018) haben viele Minister einen biografischen Hintergrund in der Regimeopposition (Fleischer 2015b). Wie dieser Beitrag allerdings gezeigt hat, ist ein hoher Opferanteil in der Regierung kein Garant für eine aktive Vergangenheitspolitik.

Ob die PT den politischen Willen und Mut hat, die von der Kommission empfohlene und vom IACHR bereits 2010 verlangte Bestrafung umzusetzen, bleibt ungewiss. Die staatlichen Akteure, die sich seit längerem für entschiedenere vergangenheitspolitische Maßnahmen (vgl. Schneider 2014) engagieren, versuchen den Bericht als Sprungbrett zu nutzen, um Druck auf die Regierung auszuüben. In einem Manifest forderten die Präsidenten der Amnestiekommission und der CEMDP, Paulo Abrão und Eugênia Gonzaga, weitreichendere Maßnahmen inklusive der Bestrafung der Täter, weitere Wahrheitskommissionen und ein politisches Maßnahmenpaket zur Stärkung der Demokratie (Ministério da Justiça 2015).

$\mathrm{Ob}$ und wie die Empfehlungen umgesetzt werden, wird zudem von zivilgesellschaftlichen Akteuren abhängen. Zweifellos haben seit 2012 neue Akteure eine klare politische Abkehr von der Militärvergangenheit gefordert. Während die Opfer lange am Rande der Gesellschaft gekämpft und die Aufklärung der Verbrechen eingefordert haben, so mobilisiert sich seit 2012 eine neue brasilianische Jugendbewegung - die sogenannte „Volksbewegung“ (Levante Popular). Sie organisiert neben Diskussionen und Kundgebungen auch Protestaktionen, um Ex-Folterknechte bloßzustellen (sogenannte escrachos ähnlich den argentinischen escraches/chilenischen funas; Kaiser 2002). Anders als die Filme und Telenovelas der 1990er, die die Diktatur größtenteils romantisiert haben, organisieren diese Akteure kritische Kulturveranstaltungen, z. B. den sogenannten Samba da Resistência (etwa: Samba als Widerstand). Zum Jahrestag des Putsches am 1. April 2015 wurden auf dem Platz der Gewaltenteilung in Brasília regimekritische Sambalieder gespielt, lautstark von Dutzenden Brasilianern mitgesungen.

Zudem haben sich in ganz Brasilien lokale, zivile Wahrheitskomitees gebildet, die eigene Berichte verfasst haben und die nun beendete brasilia- 
nische Wahrheitskommission oft zeitlich überdauern. Die Wahrheitskommission hat somit paradoxerweise - denn viele der lokalen Kommissionen entstanden aus Protest gegen die als intransparent, opferferne und passiv empfundene Nationale Wahrheitskommission - indirekt eine einzigartige Netzwerkstruktur gefördert, die eine breite Beteiligung der Zivilbevölkerung ermöglicht. Viele dieser Komitees haben Debatten organisiert und für eine breitere Reperkussion des Themas in der Öffentlichkeit gesorgt.

Trotz dieser seit 2012 zunehmenden erinnerungskulturellen Mobilisierung gab es in jüngster Zeit herbe Rückschläge. Auf mehreren Massenprotesten gegen die Regierung angesichts des Korruptionsskandals forderten Demonstranten auf Plakaten eine Militärintervention - intervenção militar já (Minstério da Justiça 2015: 9). So stehen sich scheinbar auch noch im Jahr 2015 Kritiker und Nostalgiker des Regimes unversöhnlich gegenüber, während große Teile der Bevölkerung und der Regierung Desinteresse am Thema zeigen.

Derzeit ist eine fundierte Aussage über die Rolle der Wahrheitskommission für die brasilianische Vergangenheitspolitik und Erinnerungskultur verfrüht. Zudem stellt sich die theoretische Frage, ob eine solche Bewertung eindeutig sein kann. Womöglich kann eine wissenschaftliche (nicht politische oder moralische) Evaluierung lediglich die Interpretationen der verschiedenen Interessengruppen analysieren. ${ }^{11}$

Kathryn Sikkink und Bridget Marchesi (2015) haben die brasilianische Wahrheitskommission bereits als insgesamt positiv bewertet. Sie sei „tiefgehend“, „gewillt, die Namen [der Täter] zu nennen“, und lobenswert aufgrund ihres Rufes nach Bestrafung. Die Autorinnen benutzen sogar ein vom Transitional Justice Research Collaborative-Projekt entwickeltes Benotungssystem. Während die 43 bisher abgeschlossenen Wahrheitskommissionen im Durchschnitt mit einer Punktzahl von 3,17 bis 7 bewertet wurden (7 ist die Höchstnote), geben die Autorinnen der brasilianischen Kommission die Note 6. Wenngleich ein Benotungssystem den Vergleich von Wahrheitskommissionen erleichtern mag, so würden die meisten brasilianischen Menschenrechtsexperten dieser Note widersprechen (ISER 2013; Schneider 2013; vgl. hierzu auch den Beitrag von Oettler in diesem Band). Die Opferfamilien und Überlebenden sowie deren Unterstützer (Menschenrechtsaktivisten, Staatsanwälte, Teile der Kirche) haben eine kritischere Haltung und kennen die ambivalente Geschichte der brasilianischen Vergangenheitspolitik und Erinnerungskultur im Detail. Nur zu erwähnen, wie Sikkink und Marchesi es in ihrem kurzen Beitrag

11 Der Unterschied zwischen „wissenschaftlich“ und „politisch“ ist problematisch. Siehe hierzu: Schneider (i. E.). 
Nina Schneider

tun, dass die drei letzten Präsidenten - Cardoso, Lula und Rousseff - im Gefängnis waren, verklärt den widersprüchlichen Prozess der Vergangenheitsaufarbeitung, anstatt ihn $\mathrm{zu}$ verstehen und kritisch zu analysieren. Selbst Opfer haben in hohen staatlichen Positionen paradoxerweise eine aktive Vergangenheitspolitik gebremst. Wie viele Studien beschränkt sich ihr quantitativer Ansatz zudem lediglich auf staatliche Maßnahmen, anstatt die Geschichtsmächtigkeit weiterer Akteure mit einzubeziehen und Erinnerungskultur als Ganzes zu betrachten - als eine dynamische, widersprüchliche Auseinandersetzung unterschiedlichster Interessengruppen.

\section{Literatur}

Abrão, Paulo/Torelly, Marcelo D. (2011): The Reparations Program as the Lynchpin of Transitional Justice in Brazil. In: Reátegui, Félix u. a.: Transitional Justice: Handbook for Latin America. Brasília and New York, S. 443-485.

Alves, Maria H. M. (1985): State and Opposition in Military Brazil. Austin.

Araújo, Maria Celina de (2010): Os ministros da nova república: notas para entender a democratização do poder executivo, http://cpdoc.fgv.br/artigos/, Stand: 10. Januar 2010.

Arquidiocese de São Paulo (1985): Um relato para a história. Brasil: nunca mais. Petrópolis.

Assmann, Aleida (1999): Erinnerungsräume. Formen und Wandlungen des kulturellen Gedächtnisses. München.

Assmann, Aleida/Frevert, Ute (1999): Geschichtsvergessenheit - Geschichtsversessenheit. Vom Umgang mit der deutschen Vergangenheit nach 1945. Stuttgart.

Assmann, Jan (1999): Kollektives und kulturelles Gedächtnis. Zur Phänomenologie und Funktion von Gegen-Erinnerung. In: Bosdorf, Ulrich/Grütter, Heinrich: Orte der Erinnerung. Denkmal, Gedenkstätte, Museum. Frankfurt a. M., S. 13-32.

Atencio, Rebecca J. (2014): Memory's Turn: Culture and Transitional Justice in Brazil. Madison.

Brito, Alexandra Barahona de (2013): „Justiça transicional“ em câmara lenta: o caso do Brasil. In: Pinto, Antonio Costa/Palomanes, Francisco Carlos Martinho: O passado que não passou: a sombra das ditaduras na Europa do Sul e na América Latina. Rio de Janeiro, S. 235-260.

Brito, Alexandra Barahona de (2001): Truth, Justice, Memory, and Democratization in the Southern Cone. In: Brito, Alexandra Barahona de/González-Enríquez, Carmen/ Aguilar, Paloma: The Politics of Memory: Transitional Justice in Democratizing Societies. Oxford und New York, S. 119-160.

Cornelissen, Christoph (2012): Erinnerungskulturen, Version: 2.0. In: DocupediaZeitgeschichte, 22. Oktober 2012, http://docupedia.de/zg/Erinnerungskulturen_ Version_2.0_Christoph_Corneli.C3.9Fen?oldid=106410 (Stand 1. April 2015).

Cardoso, Fernando Henrique (2006): A Arte Política: A História que viví. Rio de Janeiro. 
Eser, Patrick/Peters, Stefan (i. E.): El atentado contra Carrero Blanco como lugar de la (no-)memoria - giros entorno a un hueco en la memoria colectiva desde perspectivas interdisciplinarias. In: dies. (Hg.): El atentado contra Carrero Blanco como lugar de la (no-)memoria - Narraciones históricas y representaciones culturales. Frankfurt (Main).

Fico, Carlos (2012): Brasil: a transição inconclusa. In: Araújo, Maria Paula/Fico, Carlos/Grin, Monica: Violência na história: Memória, trauma e reparação. Rio de Janeiro, S. 25-37.

Fleischer, David (2015a): Brazil Focus Special Report, 2. Januar 2015.

Fleischer, David (2015b): Brazil Focus Special Report, 11. April 2015.

Guerreiro, G. (2009): Aprovação de Lula bate recorde histórico, diz Datafolha, http://www1.folha.uol.com.br/folha/brasil/ult96u498149.shtml (Stand 1. Oktober 2009).

Kaiser, Susana (2002): Escraches: demonstrations, communication and political memory in post-dictatorial Argentina. In: Media, Culture \& Society, 24, S. 499516.

Memórias Reveladas 2009

Mezarobba, Glenda (2013): Brazil. Stan, Lavinia und Nedelski, Nadya. Encyclopedia of Transitional Justice 3. Cambridge, S. 67-73.

Mezarobba, Glenda (2007): O preço do esquecimento: as reparações pagas às vítimas do regime militar (uma comparação entre Brasil, Argentina e Chile). Doktorarbeit, Universität São Paulo.

Ministério da Justiça (2015): Comissões de Anistia e de Mortos e Desaparecidos entregam agenda à PGR, http://www.justica.gov.br/noticias/comissoes-de-anistia-emortos-e-desaparecidos-entregam-agenda-a-pgr (Stand: 17. April 2015).

Pereira, Anthony W. (2005): Political (In)justice: Authoritarianism and the Rule of Law in Brazil, Chile, and Argentina. Pittsburgh.

Rüsen, Jörn (1994): Was ist Geschichtskultur? Überlegungen zu einer neuen Art, über Geschichte nachzudenken. In: Füssmann, Klaus/Grütter, Heinrich T./Rüsen, Jörn: Historische Faszination. Geschichtskultur heute. Köln, S. 3-26.

Santos (2015

Schneider, Nina/Atencio, Rebecca (2015): Reckoning with Dictatorship in Brazil: The Double-Edged Role of cultural-artistic Production. In: Latin American Perspectives (forthcoming).

Schneider, Nina (2014): Waiting for (an) „Apology“: Has Post-Authoritarian Brazil Apologized for State Repression? In: Journal of Human Rights, 13: 1, S. 69-84.

Schneider, Nina (2013): „Too little too late“ or „premature“? The Brazilian Truth Commission and the Question of „best timing“. In: Journal of Iberian and LatinAmerican Research, 19: 1, S. 149-162.

Schneider, Nina (2011a): Breaking the ,Silence‘ of the Military Regime: New Politics of Memory in Brazil. In: Bulletin of Latin American Research, 30: 2, S. 198-212.

Schneider, Nina (2011b): The Supreme Court's recent Verdict on the Amnesty Law: Impunity in Post-authoritarian Brazil. In: European Review of Latin American and Caribbean Studies, 90, S. 39-54.

Schneider, Nina (2011c): Truth no more? The Struggle over the National Truth Commission in Brazil. In: Iberoamericana, 42, S. 164-170. 
Nina Schneider

Schneider, Nina (2011d): Das Schlusslicht Lateinamerikas? Neueste Auseinandersetzungen über die Militärvergangenheit in Brasilien. In: Zeitschrift für Geschichtswissenschaft - ZFG, 59, S. 641-653.

Sikkink, Kathryn/Marchesi, Bridget (2015): Nothing but the Truth: Brazil's Truth Commission looks back. In: Foreign Affairs, 26. Februar 2015.

Souza, Carlos A. de (1995): Dirigente mundial da Anistia critica FHC (Fernando Henrique Cardoso). In: Folha de S. Paulo, 12. April 1995, S. 11.

Teles, Edsons/Quinalha, Renan (2015): What are the meanings of transitional justice in Brazil? In: Schneider, Nina/Esparza, Marcia: „Transitional Justice“ and the Legacies of State Violence in Latin America: A Janus-faced Paradigm? Lanham (im Erscheinen).

Wilcken, Patrick (2012): The Reckoning: Investigating Torture in Brazil. In: New Left Review, 78, S. 73-90.

Quellen

Brasil Nunca Mais Digital (BNMD) (1985, 2013): Brasil Nunca Mais, http://bnmdigital.mpf.mp.br/ (Stand: 16. April 2015).

CNV (Comissão Nacional de Verdade) (2014): Relatório Final, vol. 1-3 (Dezember 2014). Brasília.

Interviews der Autorin (2012) mit Criméia de Almeida Teles, Edson Teles und Eugênia Gonzaga sowie zwölf anonymen Überlebenden im Juli und August 2012 in São Paulo.

ISER (Instituto de Estudos da Religião) (2013): Um Ano de Comissão da Verdade: Contribuições Críticas para o Debate Público. $2^{\circ}$ Relatório de Monitoramento da Comissão Nacional da Verdade (maio de 2012 a maio de 2013). Rio de Janeiro.

Secretaria Especial dos Direitos Humanos (SEDH) (2007): Direito à memória e à verdade: comissão especial sobre mortos e desaparecidos políticos. Brasília. 